\title{
Radiological Evaluation of Orbit in cases of Proptosis in Eastern Nepal
}

\author{
R K Rauniyar ${ }^{1}$, S D Thakur ${ }^{2}$, A Panda ${ }^{2}$, U Sharma ${ }^{3}$ \\ ${ }^{1}$ Dept. of Radio-diagnosis and Medical Imaging, ${ }^{2}$ Department of Ophthalmology, B P Koirala \\ Institute of Health Sciences, Dharan, Nepal , ${ }^{3}$ B \&B Teaching Hospital Lalitpur, Nepal
}

\begin{abstract}
Aim: Proptosis is a common problem, encountered in ophthalmic outpatient department (OPD) requiring radiological evaluation for early diagnosis and to know the extent of involvement. This study was carried out to analyse the spectrum of diseases, which manifested as proptosis in eastern Nepal. Role of Computed tomography (CT) and early diagnosis is emphasised. Materials and Methods: All the patients referred to the radiology department for the evaluation of proptosis either by plain radiography, ultrasonography (USG) and CT over the period of two years were included. Results: There were 143 eyes with proptosis (including due to acute trauma), which underwent CT scan and in many of them USG and colour doppler examination were also done. Ocular and orbital tumours were most common cause of proptosis accounting for $36.36 \%$ (52) of cases followed by pseudotumour and infection $22.37 \%$ (32). Conclusion: Many of the cases of proptosis in this part of Nepal were neglected presenting late in advanced stage of disease requiring extensive surgery. This calls for early CT evaluation of the cases of proptosis and prompt management.
\end{abstract}

Key words: Computed tomography, Proptosis, Ocular and orbital tumours.

\section{Introduction}

In the past 20 years $\mathrm{CT}$ and Magnetic Resonance imaging (MRI) have emerged as the primary modalities for the evaluation of orbital diseases. One can safely say that plain radiographs have no role in the detailed evaluation of disorders of this morphological area ${ }^{1} \mathrm{CT}$ and MRI are crucial not only in the diagnostic evaluation but also in treatment planning and follow up monitoring of diseases. Hence considerable improvement and greater accuracy in diagnosis have been achieved in the field of ophthalmology by using CT.

Proptosis, due to various disorders is a common condition among the patients

Correspondence to: Dr. R. K. Rauniyar, Prof. and Head, Department of Radiodiagnosis and Imaging, BPKIHS, Dharan, Nepal

Email: rauniyar99@yahoo.com attending ophthalmic OPD. These cases require radiological evaluation for early diagnosis and appropriate medical and surgical management to improve the outcome. Advanced or neglected cases requires extensive surgery either enucleation/exenteration with poor prognosis. Clinical evaluation may provide general clue for the hidden lesion, radiological evaluation with plain radiographs, USG, CT and MRI will confirm the diagnosis. Plain radiography is of little value in evaluation of proptosis except in cases of trauma. USG is useful for ocular lesion where as CT is useful for retrobulbar lesion and to know the exact extend of the ocular and orbital lesion. Better results can be achieved if these two techniques are combined together. MRI is useful for lesion at orbital apex and optic nerve pathway but cost factor and its universal availability especially in developing countries like Nepal is a limitation. 
In this part of world (Eastern Nepal), no data is available regarding the spectrum of lesion causing proptosis. This study was carried out prospectively to analyse the lesions, which causes proptosis. Further the role of USG and CT scan in evaluation of proptosis and imaging features of various lesions are described.

\section{Materials and methods}

All the patients who were referred to radiology department for evaluation of proptosis from ophthalmic OPD over the period of two years were included in the study.

Plain radiographs and USG of orbit $(7.5 \mathrm{mHz})$ were done where required and all these patients underwent CT scan of the orbit. Diagnosis were confirmed either by characteristic imaging features of the lesions or fine needle aspiration cytology / excisional biopsy wherever possible. The results were analysed.

\section{Results}

There were 143 cases of proptosis underwent CT scan, out of which in 33 patients (Retinoblastoma-18, Malignant melanoma-2, haemangioma-10, myocysticercosis-2, carotid-cavernous-fistula-1) USG and colour doppler were diagnostic even though they also required $\mathrm{CT}$ scan to confirm and to known the exact extent of the lesion before surgery. 11 patients with trauma had plain radiographs of orbit of which 9 showed orbital fracture where as CT showed fracture of orbital wall in all patients.

The causes of proptosis in our study are given in Table 1. Among the ocular and orbital tumour (52), ocular tumours were 20 $(38.46 \%)$ and orbital tumours were 32 $(61.53 \%)$. Of these $24(46.15 \%)$ were malignant. Retinoblastoma was the commonest malignancy.(Table2)

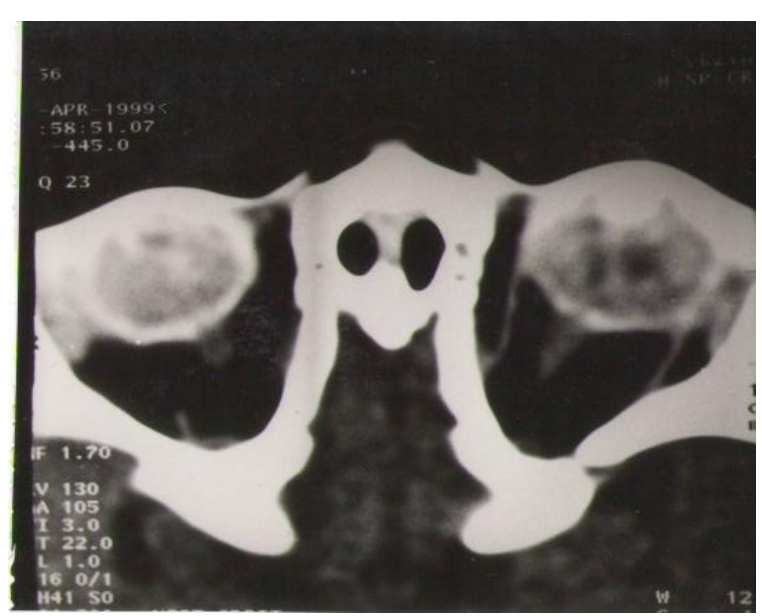

Figure 1: Bilateral retinoblastoma - Axial contrast enhanced. CT of orbit showing intraocular enhancing mass with calcification.

In our series retinoblastoma was the most common ocular tumour in infant and children. Age of the patients ranged from. 1-4 years with mean age 1.6 years and $\mathrm{M} / \mathrm{F}$ ratio was $2: 1$. In two cases retinoblastoma were bilateral (Fig. 1). Calcifications were found in 16 cases while two cases were without calcification. 13 cases showed retrobulbar and scleral extension while in 5 cases sclera was intact and in 5 cases anterior fungation and suppuration covering the entire orbit with superadded infection were seen, 7 cases there were thickened enhancing optic nerve in which one showed parasellar deposit (Fig.2). On USG heterogénous mass with echogenic foci with shadowing (calcification) was common imaging features while on CT showed intraocular enhancing mass with and without calcification.

Two cases of melanoma (45 years and 58 years) showed well-defined dome shaped intraocular mass projecting into vitreous without calcification with moderate enhancement. One of these had intact sclera while other had retrobulbar extensions. On colour doppler these lesions were highly vascular. Optic nerve glioma was the most common extra-ocular tumour in children 
causing proptosis. The age of the patient ranged from

18 months to 13 years with average age 6.7 years and M: F ratio was 4:1. The lesion was diagnosed radiologically with characteristic CT finding of fusiform enlargement involving optic nerve with mild to moderate contrast enhancement (Fig.3). In four patients widened optic canal was noted.

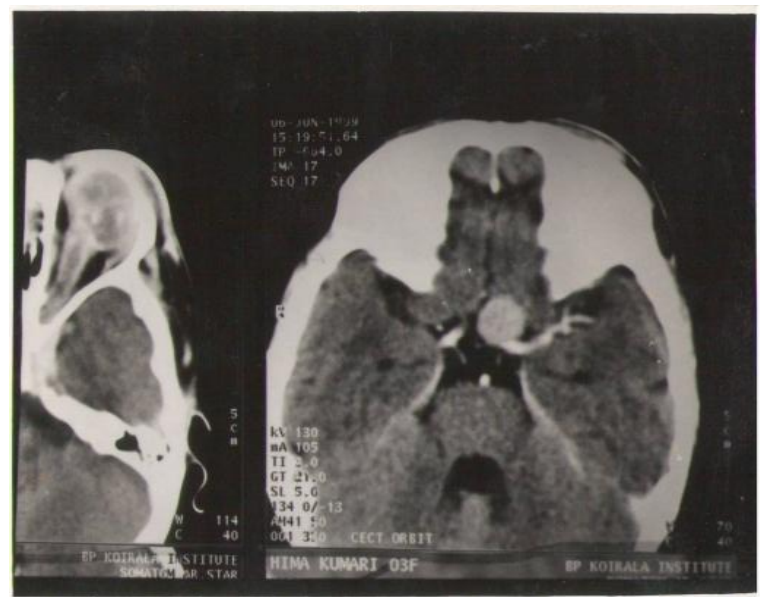

Figure 2: Intraocular mass with calcification with thickened enhancing optic nerve with parasellar deposit.

Haemangioma were most common extraocular intraorbital tumour causing proptosis in adult. The age of patient ranged from 20 to 42 years with average age 32.8 years and $\mathrm{M}$ : $\mathrm{F}$ ratio $2: 3$. On USG, these lesion were seen as well defined homogenous echotexture retrobulbar intraconal mass with variable vascularity on colour flow mapping. Well defined capsulated intraconal mild to moderately enhancing mass displacing the optic nerve were characteristic CT findings (Fig.4) except in one cases were the lesion was extraconal and medial to eye ball.

Next to haemangioma, meningioma was common cause of proptosis in adult. Intraorbital and intracranial meningioma found in 7 cases. The age ranged form 26-39 years with average age 31.2 years and $\mathrm{M}$ : F ratio was 3:4. Two cases were optic nerve sheath meningioma while others were at

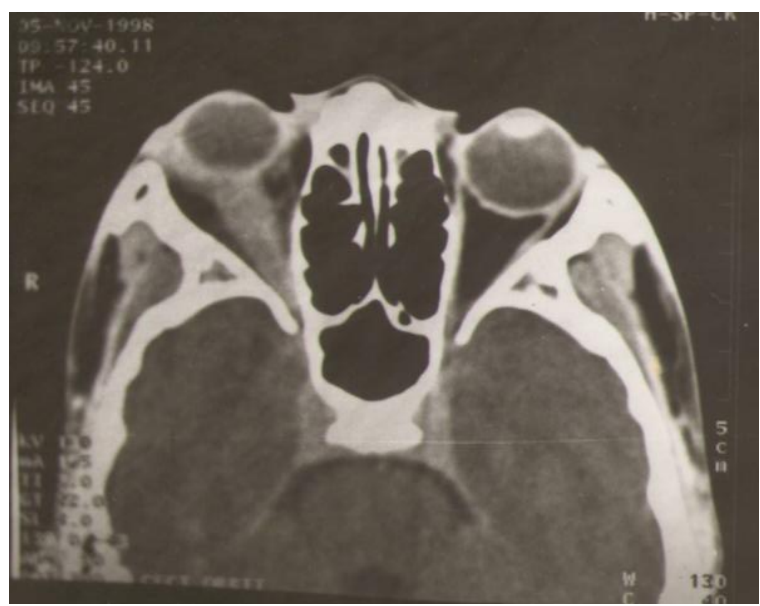

Figure 3: Optic nerve Glioma -Axial contrast enhanced CT of orbit showing fusiform enlarge of optic nerve with minimal enhancement.

petrous apex (2), parasellar and suprasellar region with orbital extension (3). On CT they appeared as well defined hyper dense mass lesion with calcification in only one of them with marked homogenous contrast enhancement. Minimal sclerosis of adjacent bones were also noted in one case.

Lacrimal gland tumour accounted for $2.79 \%$ of total cases of proptosis of which pleomorphic adenoma found in 3 cases and in one case it was lymphoma. Pleomorphic adenoma presented on CT as heterogeneously enhancing mass lesion in superolateral part of orbit with proptosis while lymphoma presented as homogenous hyper dense lesion with minimal contrast enhancement (Fig. 5).

Proptosis due to metastasis was found in 3 cases (3/143) accounting for $2.09 \%$ of total cases of proptosis. One case bilateral orbital metastasis in 3 years female was from neuroblastoma, others were from renal cell carcinoma and from melanoma.

\section{Proptosis due to adjacent PNS lesion}


In present study $20.97 \%$ of cases of proptosis were due to orbital spread of adjacent paranasal sinuses lesion. (Table 3)

Nine cases of nasopharyngeal carcinoma proptosis were due to involvement of cavernous sinuses and orbital extension. In 5 cases there was bony destruction at base of skull with intracranial extension. Unilateral orbital extensions were in 4 cases while extension in bilateral orbit was in 5 cases. Age of the patient ranged from 35- 60 years with average age 38 years and M: F ratio was 4:5. On CT they appeared as enhancing mass on roof and lateral wall of nasopharynx with bony destruction and orbital extension. In one patient

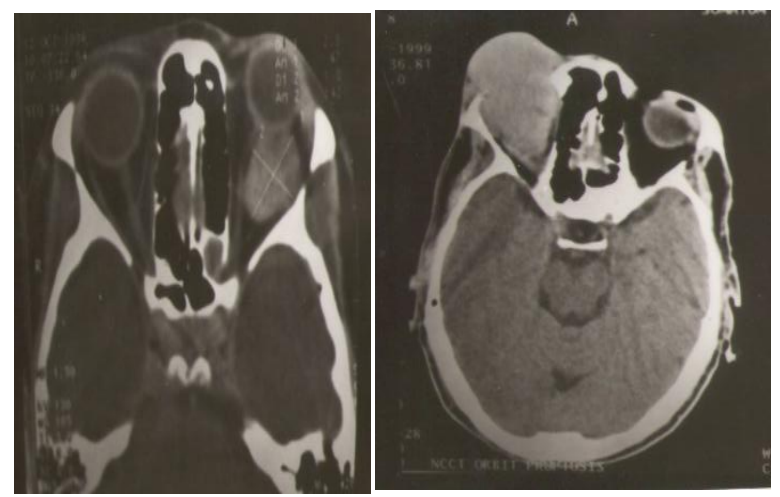

Figure 4(left): Haemangioma left orbit Axial CECT of orbit showing intraconal mildly enhancing oval mass displacing the optic nerve. Figure 5(Right): Lymphoma of lacrimal gland- Axial plain CT of orbit showing homogenous hyperdense mass in right orbit which showed minimal enhancement on post contrast study.

orbital extension was from inferior orbital fissure and in one through destruction of medial wall of orbit.

Carcinoma of maxillary sinus accounted for $5.59 \%$ of total cases of proptosis, 5 cases were in right side while 3 were in left side with age group ranged from 40-52 years with average age 42 years and $\mathrm{M}$ : $\mathrm{F}$ ratio was 5:3. All cases showed bony destruction in roof of maxillary sinus with orbital extension (Fig.6). 5 cases showed extension into nasal cavity and one showed extension into contralateral nasal cavity with destruction of nasal septum. All the cases histologically were squamous cell carcinoma.

Squamous cell carcinoma of ethmoid with orbital extension with Proptosis accounted for $4.19 \%$ of total cases of proptosis. The age varied from 28-45 years with average age 40 years with M: F ratio 3:2. Two cases showed intracranial extension.

Benign lesion of frontal sinus mucocele and pyocele caused proptosis in 3 cases due to orbital extension.

In our series $7.69 \%(11 / 143)$ of cases proptosis was due to acute trauma and foreign body. These cases were fracture of orbital wall with retrobulbar haematoma with associated head injury (5), fracture of floor of orbit (2), foreign body (2), orbital apex fracture (1), haematoma with orbital emphysema (1).

\section{Pseudotumour and infection}

This group of lesion accounted for $22.37 \%$ (32) total cases of proptosis. Among these $8.39 \%$ (12) were due to retrobulbar cellulites with abscess and $13.98 \%$ (20) was due to pseudotumour. The age of the patient with Pseudotumour ranged from 9 years to 51 years with average age 30 years with M: F:: $3: 2$. All the cases of pseudotumour were of diffuse type involving muscle, retrobulbar space, optic nerve, lacrimal gland and peribulbar region except in four cases where isolated muscle involvement (myositis) was found. All these patients with Pseudotumour responded well with steroid with compleate resolution of lesion on follow up CT.

Proptosis in 12 cases was due to orbital cellulitis and or abscess. Four cases were abscess one inferior to eyeball other in retrobulbar location with thick irregular wall with CT density ranging from 15-20 Hf required surgical drainage, while cellulitis 
responded to antibiotics. Six of them had purulent sinusitis.

\section{Miscellaneous Group}

This group consisted of proptosis due to Graves' disease (5), myxoid tumour (2), parasitic cysticercosis (5), caroticocavernous fistula (CCF) (2), lymphoma of eyelid (1), tumour of neural origin (2), and enchephalocele extending into orbit with proptosis (1). All the cases of Graves' disease

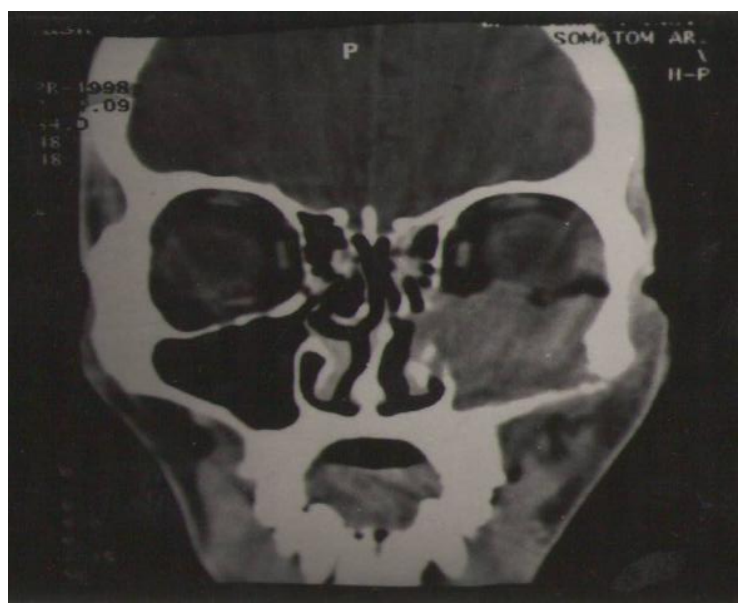

Figure 6: Carcinoma of right maxillary sinus with orbital extension with bony destruction.

showed characteristic thickening of belly of extraocular muscle which was bilateral in 4 cases and unilateral in one.

\section{Discussion}

Proptosis is one of the puzzling presentations in the ophthalmology department. A wide variety of diseases ranging from infective to neoplastic may present with proptosis.

Sometimes the group of clinical features lead to the diagnosis easily; but sometimes they leave the clinician confused. The radiologist has an important role to play not only to help in confirming the diagnosis but also to know the extent of involvement.
CT has become accepted as the key modality in orbital radiography ${ }^{2}$. Good technique with axial and coronal positioning on high resolution scanners provides excellent imaging of major orbital structures including the globe, ocular muscles, optic nerve and major vessels.

In a study conducted by Handler et $\mathrm{al}^{3}$ on 65 patients suffering from acute inflammatory disease of the orbit, orbital ceihilitis was diagnosed in 17 and subperiosteal abscess in the remaining 48. In our study of 143 patients orbital cellulitis was diagnosed in 12 patients.

The preferred radiologic method used for assessment of pseudotumour is $\mathrm{CT}^{4}$. The clinical and radiological constellation of findings allows a definitive diagnosis in most cases. In cases of Pseudotumour radiological findings are grouped according to location in to dacryadenitis, myositis and sclerouveitis with and without associated infiltration. Further they have also highlighted that MRI is more useful than CT in orbital apex pathology as Tolosa-hunt syndrome which is characterised by an inflammatory infiltrate in the orbital apex and cavernous sinuses leading to cranial nerve involvement with ophthalmoplegia. Orbital pseudotumours account for approximately $8 \%$ of the orbital tumours 5 . Pseudotumour is the third most common ophthalmologic disease after Graves' disease and lymphoproliferative disease $^{6}$. The imaging findings correlated with clinical findings, allow a diagnosis is most cases and hence, obviate the need for a biopsy. But in our study, Pseudotumour accounted for $13.98 \%$ (20) of total cases of Proptosis where all of the cases were diffuse type involving muscle, retrobulbar space, optic nerve lacrimal gland except in four where isolated myositis type was found. In all our patients clinical and radiological finding were confirmatory and hence non of them required biopsy and responded well with steroid with complete resolution in follow up CT. 
Graves' disease is the most common orbital disorder affecting approximately $0.5 \%$ of the population in $\mathrm{USA}^{7}$. It is the underlying causes in $15 \%-28 \%$ of cases of unilateral exophthalmos and in $80 \%$ cases with bilateral exophthalmos. In our study there were 5 patients with Graves' disease .of which four had bilateral and one had unilateral involvement. This low figure is probably due to the fact that in most of the patients diagnosis was clinically evident and CT was not done.

Accurate evaluation of orbital tumours is most important for therapeutic management. Tissue diagnosis of orbital tumours is especially difficult because of the wide range of tissues normally occurring in the orbit. Unfortunately, such tumours are relatively surgically inaccessible for biopsy due to presence of bony walls. This gives rise to a need for effective methods of indirect assessment of the orbit prior to definitive therapy. USG, CT and MRI are the modality of choice in the assessment of orbital neoplasia but each has its own limitation. This enables the clinician to delineate the extent of disease and plan the surgical approach for biopsy and excision. In some cases serial orbital imaging is used to monitor for diseases status.

Rodahl $\mathrm{E}$ et $\mathrm{al}^{8}$ retrospectively evaluated the records of 278 patients with orbital tumours, of these 177 patients had benign tumours and 96 patients had malignant tumours. In our study, there were 52 patients with ocular and orbital tumours of these 28 patients had benign neoplasms while 24 patients had malignant neoplasms which is almost similar to the incidence reported by Rodahl E et $1^{8}$.

Mullaney PB et $\mathrm{al}^{9}$ evaluated record of 292 retinoblastoma patients and found that 14 patients with retinoblastoma had associated cellulitis $(4.8 \%)$ which was not indicative of an extension of retinoblastoma into the orbit. In fact they highlighted that radiological evaluation of scleral integrity may be hindered by periocular inflammatory changes. In our series CT clearly showed retrobulbar and scieral extension in 13 patients without any evidence of cellulites while in 5 cases with fungation and suppuration CT clearly showed evidence of infection.

Complex orbital rim and fractures of the internal orbital skeleton (orbital walls) are best analysed by high resolution axial CT. MRI usually is not the initial modality for assessment of orbital trauma, but it is helpful in evaluating vascular injuries such as carotid cavernous fistula or post traumatic pseudoaneurysms $^{10}$. Our series had 11 patients with orbital trauma where fractures were clearly delineated on CT where as plain radiographs failed to identify fracture in two patients. Moreover CT clearly depicted status of orbital soft tissue in relation to fracture. Hence we believe CT is much more superior in case of trauma of the orbit.

Early CT scan evaluation is a must, as over $33 \%$ of patients in our series required prompt and adequate medical therapy and another $20 \%$ required otolaryngiologist's intervention. The value of CT scan lies in its ability to demonstrate haematoma, calcification, fracture, bone destruction, and tumours; and to define their orbital and extra orbital extension and their relationship to neighbouring structure.

Koeller KK et $a^{11}$ introduced "space approach" as a means of analysing orbital masses on imaging studies. Determination in which space an orbital mass resides and its other features allows the radiologist to narrow the differential diagnosis and provides essential information for definitive diagnosis and treatment planning. We do agree this is useful way for the diagnosis where biopsy is not possible.

CT scan is an invaluable tool in confirming the diagnosis when the clinical presentation is not classical; and to know the extent of involvement in cases of neoplasia to plan the surgery accordingly. 
Table 1 -Total cases (143)

\begin{tabular}{ll}
\hline Diagnosis & No. (\%) \\
\hline Ocular and orbital tumour & 52 \\
& $(36.36 \%)$ \\
Pseudotumour and infection & 32 \\
& $(22.37 \%)$ \\
\hline $\begin{array}{l}\text { Proptosis due to adjacent PNS } \\
\text { lesion }\end{array}$ & 30 \\
Trauma including foreign body & 11 \\
& $(7.69 \%)$ \\
Miscellaneous (Graves disease, & 18 \\
CCF, Parasitic infestation & $(12.58 \%)$ \\
Enchephalocele etc) & \\
\hline
\end{tabular}

Table 2- Ocular and orbital tumour (52)

\begin{tabular}{ll}
\hline Diagnosis & No. $(\%)$ \\
\hline Retinoblastoma & $18(34.61 \%)$ \\
Malignant melanoma & $2(3.84 \%)$ \\
Optic nerve glioma & $8(15.38 \%)$ \\
Haemangioma & $10(19.23)$ \\
Meningioma & $7(13.46 \%)$ \\
Lacrimal gland tumour & $4(7.69 \%)$ \\
Metastasis & $3(5.76 \%)$ \\
\hline
\end{tabular}

Table 3: Adjacant sinus lesions causing proptosis ( 30 cases)

\begin{tabular}{ll}
\hline Diagnosis & No. (\%) \\
\hline $\begin{array}{l}\text { Nasopharyngeal carcinoma } \\
\text { Carcinoma of maxillary } \\
\text { sinus }\end{array}$ & $9(30 \%)$ \\
$\begin{array}{l}\text { Carcinoma of ethmoid } \\
\text { sinus }\end{array}$ & $6(24.66 \%)$ \\
$\begin{array}{l}\text { Frontal sinus mucocele / } \\
\text { pyocele } \\
\text { Antral polyp }\end{array}$ & $3(10 \%)$ \\
$\begin{array}{l}\text { Nasopharyngeal } \\
\text { angiofibroma }\end{array}$ & $1(3.33 \%)$ \\
$\begin{array}{l}\text { Fungal mass (Ethnoid, } \\
\text { Maxillary and sphenoid) }\end{array}$ & $1(3.33 \%)$ \\
\hline
\end{tabular}

\section{References}

1. Belden CJ, Zinreich SJ. Orbital imaging techniques. Semin. Ultrasound CT MR 1997; 18:413-22.
2. Forbes G. Computed tomography of the orbit. Radiol din North Am 1982; 20:37-47.

3. Handler LC, Davey LC, Hill JC, Lauryssen C. The acute orbit: differentiation of orbital cellulitis from subperiosteal abscess by computerised tomography. Neuroradiology 1991; 33: 15-8.

4. Weber AL, Romo LV, Sabates NR. Pseudotumour of the orbit. Clinical, pathologic and radiologic evaluation. Radiol Clin North Am 1999; 37:15168.

5. Groutean E, Chaix U, Arubruster V. Lesneur L, Sevely A, Rubie H et al. Acute orbital myositis and idiopathic inflammatory pseudoturnoür in children: 3 cases. Arch Pediatr 1998; 5: $153-8$

6. Weber AL, Jakobiec FA, Sabates NR. Pseudotumour of the orbit. Neuroimaging Clin N Am 1996; 6:7392.

7. Weber AL, Dallow RL, Sabates NR. Graves disease of the orbit. Neuroimaging chin N Am 1996; 6:6172.

8. Rodahl E, Bertelsen E, Seland J. Ames JB, Mork S. Orbital tumours. Tiddsskr Nor Laegeforen 2000; 120:3518-23.

9. Mullaney PB, Karcioglu ZA, Huaman AM, Al-mesfers. Retinoblastoma associated with orbital cellulitis. Br. J. Ophthalmol 1 998; 82 :517-21.

10. MaurielloJA Jr, Lee HJ, Nguyen L. CT of soft tissue injury and orbital fractures. Radiol. Clin. North Am. 1999;

37:241-52.

11. Koeller KK, Smirniotopoulos JO. Orbital masses. Semin. ultrasound CT MR. 1998;19:272- 91. 